\title{
Critical sets, crowns and local maximum independent sets
}

\author{
Vadim E. Levit \\ Department of Computer Science \\ Ariel University, Israel \\ levitv@ariel.ac.il \\ Eugen Mandrescu \\ Department of Computer Science \\ Holon Institute of Technology, Holon, Israel \\ eugen_m@hit.ac.il
}

\begin{abstract}
A set $S \subseteq V(G)$ is independent (or stable) if no two vertices from $S$ are adjacent, and by $\operatorname{Ind}(G)$ we mean the set of all independent sets of $G$.

A set $A \in \operatorname{Ind}(G)$ is critical (and we write $A \in \operatorname{CritIndep}(G)$ ) if $|A|-|N(A)|=$ $\max \{|I|-|N(I)|: I \in \operatorname{Ind}(G)\}$ [54], where $N(I)$ denotes the neighborhood of $I$.

If $S \in \operatorname{Ind}(G)$ and there is a matching from $N(S)$ into $S$, then $S$ is a crown [1], and we write $S \in \operatorname{Crown}(G)$.

Let $\Psi(G)$ be the family of all local maximum independent sets of graph $G$, i.e., $S \in \Psi(G)$ if $S$ is a maximum independent set in the subgraph induced by $S \cup N(S)$ [28.

In this paper we show that $\operatorname{CritIndep}(G) \subseteq \operatorname{Crown}(G) \subseteq \Psi(G)$ are true for every graph. In addition, we present some classes of graphs where these families coincide and form greedoids or even more general set systems that we call augmentoids.

Keywords: critical set, crown, local maximum independent set, matching, bipartite graph, König-Egerváry graph, greedoid.
\end{abstract}

\section{Introduction}

Throughout this paper $G$ is a finite simple graph with vertex set $V(G)$ and edge set $E(G)$. If $X \subseteq V(G)$, then $G[X]$ is the subgraph of $G$ induced by $X$. By $G-W$ we mean either the subgraph $G[V(G)-W]$, if $W \subseteq V(G)$, or the subgraph obtained by deleting the edge set $W$, for $W \subseteq E(G)$. The neighborhood $N(v)$ of a vertex $v \in V(G)$ is the set $\{w: w \in V(G)$ and $v w \in E(G)\}$, while the closed neighborhood $N[v]$ of $v \in V(G)$ is the set $N(v) \cup\{v\}$; in order to avoid ambiguity, we use also $N_{G}(v)$ instead of $N(v)$. The neighborhood $N(A)$ of $A \subseteq V(G)$ is $\{v \in V(G): N(v) \cap A \neq \emptyset\}$, and $N[A]=N(A) \cup A$. We may also use $N_{G}(A)$ and $N_{G}[A]$, when referring to neighborhoods in a graph $G$. A vertex $v$ is isolated if $N(v)=\emptyset$. Let us define isol $(G)$ as the set of all isolated vertices. If $A, B \subset V(G), A \cap B=\emptyset$, then by $(A, B)$ is denoted the set $\{a b: a b \in E(G), a \in A, b \in B\}$.

The graph $G$ is unicyclic if it is connected and has a unique cycle.

Let $V(G)=\left\{v_{i}: 1 \leq i \leq n\right\}$. Joining each $v_{i}$ to all the vertices of a copy of a graph $H$, we obtain a new graph, called the corona of $G$ and $H$, denoted by $G \circ H$. 
A set $S \subseteq V(G)$ is independent (or stable) if no two vertices from $S$ are adjacent, and by $\operatorname{Ind}(G)$ we mean the family of all the independent sets of $G$. An independent set of maximum size is a maximum independent set of $G$, and the independence number $\alpha(G)$ of $G$ is $\max \{|S|: S \in \operatorname{Ind}(G)\}$. Let $\Omega(G)$ denote the family of all maximum independent sets, and $\operatorname{core}(G)=\bigcap\{S: S \in \Omega(G)\}$ [18, 27]. The problem of whether core $(G) \neq \emptyset$ is NP-hard [6].

A matching in a graph $G$ is a set of edges $M \subseteq E(G)$ such that no two edges of $M$ share a common vertex. A maximum matching is a matching of maximum cardinality. By $\mu(G)$ is denoted the cardinality of a maximum matching. A matching is perfect if it saturates all the vertices of the graph. A matching $M=\left\{a_{i} b_{i}: a_{i}, b_{i} \in V(G), 1 \leq i \leq k\right\}$ is a uniquely restricted matching if $M$ is the unique perfect matching of $G\left[\left\{a_{i}, b_{i}: 1 \leq i \leq k\right\}\right]$ [17.

Recall that if $\alpha(G)+\mu(G)=|V(G)|$, then $G$ is a König-Egerváry graph [12, 53]. As a wellknown example, each bipartite graph is a König-Egerváry graph as well. Various properties of König-Egerváry graphs can be found in 5, 20, 21, 23, 29, 31, 32, 34, 42, 47.

If $S$ is an independent set of a graph $G$ and $A=V(G)-S$, then we write $G=S * A$. Evidently, each graph admits such representations. For instance, if $E(A)=\emptyset$, then $G=S * A$ is bipartite; if $H$ is complete, then $G=S * A$ is a split graph.

Theorem 1.1 [27] Let $G$ be a connected graph. Then the following are equivalent:

(i) $G$ is a König-Egerváry graph;

(ii) $G=S * A$, where $S \in \Omega(G)$ and $|S| \geq \mu(G)=|A|$;

(iii) $G=H_{1} * H_{2}$, where $V\left(H_{1}\right)=S$ is independent, $|S| \geq\left|V\left(H_{2}\right)\right|$, and $\left(S, V\left(H_{2}\right)\right)$ contains a matching $M$ with $|M|=\left|V\left(H_{2}\right)\right|$.

Let us notice that a disconnected graph is a König-Egerváry graph if and only if each of its connected components induces a König-Egerváry graph.

Theorem 1.2 [41] For a graph $G$ the following properties are equivalent:

(i) $G$ is a König-Egerváry graph;

(ii) there is $S \in \Omega(G)$ such that each maximum matching of $G$ is contained in $(S, V(G)-S)$;

(iii) for every $S \in \Omega(G)$ each maximum matching of $G$ is contained in $(S, V(G)-S)$.

For $X \subseteq V(G)$, the number $|X|-|N(X)|$ is the difference of $X$, denoted $d(X)$. The critical difference $d(G)$ is $\max \{d(X): X \subseteq V(G)\}$. The number $\max \{d(I): I \in \operatorname{Ind}(G)\}$ is the critical independence difference of $G$, denoted $i d(G)$. Clearly, $d(G) \geq i d(G)$. It was shown in [54] that $d(G)=i d(G)$ holds for every graph $G$. If $A$ is an independent set in $G$ with $d(X)=d(G)$, then $A$ is a critical independent set. A maximum critical independent set is a critical independent set of maximum cardinality. Let

$$
\begin{aligned}
\text { CritIndep }(G) & =\{S: S \text { is a critical independent set in } G\} \text { and } \\
\text { MaxCritIndep }(G) & =\{S: S \text { is a maximum critical independent set in } G\} .
\end{aligned}
$$

For example, consider the graph $G$ from Figure 1. Note that $X=\left\{v_{1}, v_{2}, v_{3}, v_{4}\right\}$ is a critical set, since $N(X)=\left\{v_{3}, v_{4}, v_{5}\right\}$ and $d(X)=1=d(G)$, while $S=\left\{v_{1}, v_{2}, v_{3}, v_{6}, v_{7}\right\}$ is a critical independent set, because $d(S)=1=d(G)$. Notice that $\left\{v_{1}, v_{2}, v_{3}, v_{6}, v_{7}, v_{10}\right\} \in$ $\operatorname{Max} \operatorname{CritIndep}(G)$. Other critical sets are $\left\{v_{1}, v_{2}\right\},\left\{v_{1}, v_{2}, v_{3}\right\},\left\{v_{1}, v_{2}, v_{3}, v_{4}, v_{6}, v_{7}\right\}$.

Critical independent sets are of interest for both practical and theoretical purposes. Zhang proved that a critical independent set can be found in polynomial time 54. A simpler algorithm, reducing the critical independent set problem to computing a maximum independent set in a bipartite graph is given in 2 . 
$G$

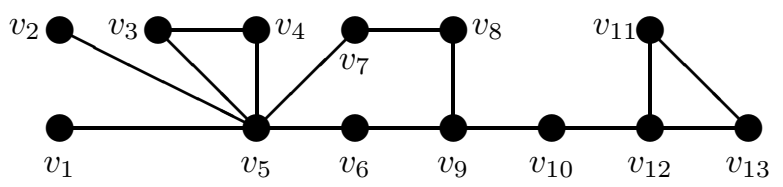

Figure 1: $\operatorname{core}(G)=\left\{v_{1}, v_{2}, v_{6}, v_{10}\right\}$ is a critical set.

Lemma 1.3 [25] There is a matching from $N(S)$ into $S$ for every critical independent set $S$.

A proof of a conjecture of Graffiti.pc [10] yields a new characterization of König-Egerváry graphs: these are exactly the graphs, where there exists a critical maximum independent set [26.

It is known that $d(G) \geq \alpha(G)-\mu(G)$ holds for every graph [36, 46, 50. In [37] it is proved the following.

Theorem 1.4 [37] For a König-Egerváry graph $G$ the following equalities hold

$$
d(G)=|\operatorname{core}(G)|-|N(\operatorname{core}(G))|=\alpha(G)-\mu(G) .
$$

Using this finding, we have strengthened the characterization from [26].

Theorem 1.5 [37] $G$ is a König-Egerváry graph if and only if each of its maximum independent sets is critical.

For a graph $G$, let us denote

$$
\operatorname{ker}(G)=\bigcap\{A: A \text { is a critical independent set }\} \text { [36]. }
$$

Clearly, isol $(G) \subseteq \operatorname{ker}(G)$. Some more on the internal structure of $\operatorname{ker}(G)$ may be found in [40].

Theorem 1.6 [36] (i) For every graph $G, \operatorname{ker}(G) \subseteq \operatorname{core}(G)$.

[39] (ii) If $G$ is a bipartite graph, then $\operatorname{ker}(G)=\operatorname{core}(G)$.

[43] (iii) If $G$ is a unicyclic non-König-Egerváry graph, then $\operatorname{ker}(G)=\operatorname{core}(G)$.

Since $|\operatorname{ker}(G)| \neq 1$ holds for any graph $G$ without isolated vertices [45, it follows that if $|\operatorname{isol}(G)| \neq 1$, then $|\operatorname{core}(G)| \neq 1$ for bipartite graphs [19, 27] and unicyclic non-KönigEgerváry graphs $[38,43$.

Notice that there are non-bipartite graphs enjoying the equality $\operatorname{ker}(G)=\operatorname{core}(G)$; e.g., the graphs from Figure 2, where only $G_{1}$ is a König-Egerváry graph.
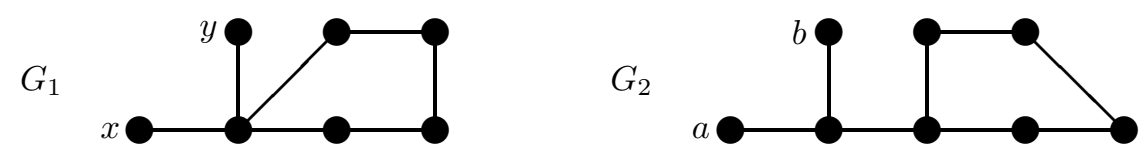

Figure 2: $\operatorname{core}\left(G_{1}\right)=\operatorname{ker}\left(G_{1}\right)=\{x, y\}$ and $\operatorname{core}\left(G_{2}\right)=\operatorname{ker}\left(G_{2}\right)=\{a, b\}$. 

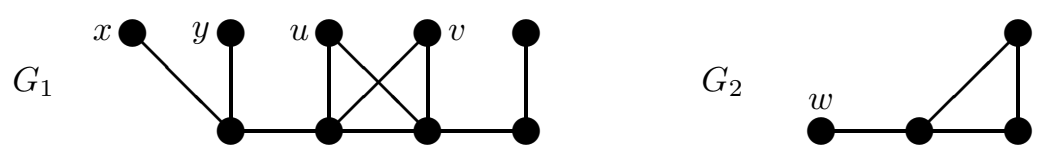

Figure 3: Both $G_{1}$ and $G_{2}$ are König-Egerváry graphs. Only $G_{2}$ has a perfect matching.

There is a non-bipartite König-Egerváry graph $G$, such that $\operatorname{ker}(G) \neq \operatorname{core}(G)$. For instance, the graph $G_{1}$ from Figure 3 has $\operatorname{ker}\left(G_{1}\right)=\{x, y\}$, while core $\left(G_{1}\right)=\{x, y, u, v\}$. The graph $G_{2}$ from Figure 3 has $\operatorname{ker}\left(G_{2}\right)=\emptyset$, while core $\left(G_{2}\right)=\{w\}$.

Recall that if $S \in \operatorname{Ind}(G)$ and there is a matching from $N(S)$ into $S$, then $S$ is a crown in $G$, of order $|S|+|N(S)|$ [1, 9, 13. If $|S|=|N(S)|$, then $S$ is a straight crown. A crown of maximum cardinality is a maximum crown. Let

$$
\begin{aligned}
& \text { Crown }(G)=\{S: S \text { is a crown of } G\} \text { and } \\
& \operatorname{Max} \operatorname{Crown}(G)=\{S: S \text { is a maximum crown of } G\} \text {. }
\end{aligned}
$$

It is convenient to consider that $\emptyset \in \operatorname{Crown}(G)$, and also $\{v\} \in \operatorname{Crown}(G)$ for every isolated vertex $v$.

Theorem 1.7 [1] Isolating a crown of maximum order is solvable in polynomial time.

Let us emphasize the significance of the above theorem in the context of the following result.

Theorem 1.8 [52] Given a graph $G$ and an integer $k$, it is $\mathbf{N P}$-complete to decide whether $G$ contains a crown whose order is exactly $k$.

A set $A \subseteq V(G)$ is a local maximum independent (stable) set of $G$ if $A$ is a maximum independent set in the subgraph $G[N[A]][28$. By $\Psi(G)$ is denoted the set of all local maximum independent sets of the graph $G$. Clearly, $\Omega(G) \subseteq \Psi(G)$ holds for every graph.

Theorem 1.9 [48] Every local maximum independent set of a graph is a subset of a maximum independent set.

Lemma 1.10 [33] If $G$ is a König-Egerváry graph, $S \in \Psi(G)$, and $H=G[N[S]]$ is also a König-Egerváry graph, then every maximum matching of $H$ can be enlarged to a maximum matching in $G$.

A greedoid is a set system generalizing the notion of a matroid.

Definition 1.11 [4], 24] $A$ greedoid is a pair $(E, \mathcal{F})$, where $\mathcal{F} \subseteq 2^{E}$ is a non-empty set system satisfying the following conditions:

Accessibility: for every non-empty $X \in \mathcal{F}$ there is an $x \in X$ such that $X-\{x\} \in \mathcal{F}$;

Exchange: for $X, Y \in \mathcal{F},|X|=|Y|+1$, there is an $x \in X-Y$ such that $Y \cup\{x\} \in \mathcal{F}$.

It is worth observing that if $\Psi(G)$ is a greedoid and $S \in \Psi(G),|S|=k \geq 2$, then by accessibility property, there is a chain

$$
\emptyset \subset\left\{x_{1}\right\} \subset\left\{x_{1}, x_{2}\right\} \subset \cdots \subset\left\{x_{1}, \ldots, x_{k-1}\right\} \subset\left\{x_{1}, \ldots, x_{k-1}, x_{k}\right\}=S,
$$


such that $\left\{x_{1}, x_{2}, \ldots, x_{j}\right\} \in \Psi(G)$, for all $j \in\{1, \ldots, k-1\}$. Such a chain is called an accessibility chain of $S$.

There are some known sufficient conditions on the graph $G$ that ensure the family $\Psi(G)$ to be a greedoid on the vertex set of $G$.

Theorem 1.12 [28] (i) If $G$ is a forest, then $\Psi(G)$ is a greedoid.

[30] (ii) For a bipartite graph $G, \Psi(G)$ is a greedoid if and only if all its maximum matchings are uniquely restricted.

[33] (iii) Let $G$ be a triangle-free graph. Then $\Psi(G)$ is a greedoid if and only if all maximum matchings of $G$ are uniquely restricted and the closed neighborhood of every local maximum independent set of $G$ induces a König-Egerváry graph.

Recall that $G$ is a well-covered graph if all its maximal independent sets are of the same cardinality [49, and $G$ is very well-covered if, in addition, it has no isolated vertices and $|V(G)|=2 \alpha(G)[14$.

Theorem 1.13 Let $G$ be a very well-covered graph. Then

(i) [32] $G[N[S]]$ is a König-Egerváry graph, for every $S \in \Psi(G)$;

(ii) [35] an independent set $S$ belongs to $\Psi(G)$ if and only if $|S|=|N(S)|$.

(iii) [35] $\Psi(G)$ is a greedoid if and only if $G$ has a unique maximum matching.

One of the most useful tool for the efficient optimization is the greedy algorithm. Its essence lies in trying to find the global optimum by moving on each step in the locally optimal direction. In particular, it resembles the classical 'gradient' search method in continuous case. If the number of steps and the complexity of choosing the local optimum are both polynomial, then, obviously, the greedy algorithm produces its result in polynomial time.

For some classes of problems, the greedy algorithm produces an optimal result. For instance, Prim and Kruskal algorithms for finding a minimum spanning tree in a graph are both greedy. In general many greedy problems can be described using exchange structures called matroids, which were later generalized to greedoids. One of the important tasks of combinatorial optimization theories is to explain the correctness of the greedy approach in those cases and to generalize them to every possible extent.

Another important task is the inverse - to define the boundaries of applicability of the greedy algorithm. It is known that every linear objective function can be optimized by a greedy algorithm on matroids, and conversely, the Rado-Edmonds theorem claims that this property characterizes matroids for hereditary set systems. In contrast with this, optimizing a linear function on greedoids is an intractable problem. On the other hand, it was shown that Gaussian greedoids allow a generalization of the Rado-Edmonds theorem [3, 4, 16, 51. In addition, there is an algorithmic characterization of antimatroids [7, 22].

In what follows, we coin an idea of a more general exchange structure that we call an augmentoid.

Definition 1.14 An augmentoid is a pair $(E, \mathcal{F})$, where $\mathcal{F} \subseteq 2^{E}$ is a non-empty family of sets satisfying the following condition:

Augmentation: for $X, Y \in \mathcal{F}$, there exist $A \subseteq X-Y$ and $B \subseteq Y-X$ such that $Y \cup A, X \cup B \in \mathcal{F}$ and $|Y \cup A|=|X \cup B|$.

Every element of $\mathcal{F}$ is a feasible set of the augmentoid $(E, \mathcal{F})$. 
Proposition 1.15 If $(E, \mathcal{F})$ is an augmentoid, then every feasible set may be enlarged to a maximum feasible set.

Proof. Let $X, Y \in \mathcal{F}$, where $Y$ is a maximum feasible set. By the augmentation property, there is a possibility to enlarge $X$ to a feasible set $X \cup B$ in such a way that $|X \cup B|=$ $|Y \cup \emptyset|=|Y|$.

Clearly every greedoid is an augmentoid. As we mentioned before, greedoids were invented in order to accommodate greedy algorithms with exchange properties. The main purpose of this paper is to extend the area of applicability of exchange structures to a more general context including critical sets, crowns, and local maximum independent sets.

\section{Preliminary results}

Let us notice that if $S$ is a crown of a graph $G$, having $M$ as a matching from $N(S)$ into $S$, then $|S|+|M|=|S|+|N(S)|=|S \cup N(S)|$. Taking into account Theorems 1.1, 1.2, one may immediately conclude with the following.

Corollary 2.1 If $S$ is a crown of a graph $G$, then $G[N[S]]$ is a König-Egerváry graph.

Consequently, if $S$ is a straight crown, then $G[N[S]]$ is a König-Egerváry graph with a perfect matching.
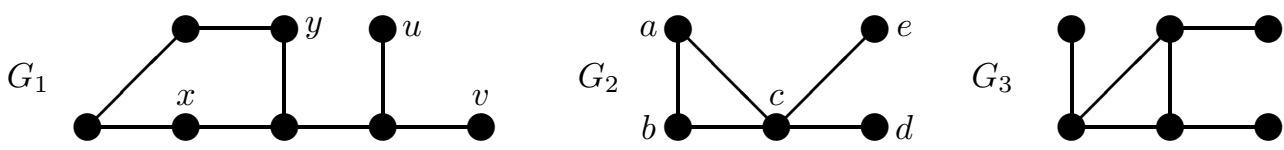

Figure 4: Only $G_{2}$ and $G_{3}$ are König-Egerváry graphs.

Let us consider the graphs from Figure 4 Notice that:

- CritIndep $\left(G_{1}\right) \neq \operatorname{Crown}\left(G_{1}\right) \neq \Psi\left(G_{1}\right)$, because $\{v\} \in \operatorname{Crown}\left(G_{1}\right)-\operatorname{CritIndep}\left(G_{1}\right)$ and $\{x, y\} \in \Psi\left(G_{1}\right)-\operatorname{Crown}\left(G_{1}\right)$;

- $\operatorname{CritIndep}\left(G_{2}\right) \neq \operatorname{Crown}\left(G_{2}\right) \neq \Psi\left(G_{2}\right)$, since $\{d\} \in \operatorname{Crown}\left(G_{2}\right)-\operatorname{CritIndep}\left(G_{2}\right)$ and $\{a\} \in \Psi\left(G_{2}\right)-\operatorname{Crown}\left(G_{2}\right)$;

- CritIndep $\left(G_{3}\right)=\operatorname{Crown}\left(G_{3}\right)=\Psi\left(G_{3}\right)$.

Theorem 2.2 CritIndep $(G) \subseteq$ Crown $(G) \subseteq \Psi(G)$ hold for every graph $G$.

Proof. Let $S \in$ CritIndep $(G)$. By Lemma 1.3, there exists a matching from $N(S)$ into $S$. Consequently, $S$ is a crown of $G$. Hence we infer that $\operatorname{CritIndep}(G) \subseteq \operatorname{Crown}(G)$.

Let now $S \in \operatorname{Crown}(G)$. Then $G[S \cup N(S)]$ is a König-Egerváry graph, by Corollary 2.1. Further, according to Theorem 1.1 (ii), $S$ is a maximum independent in $G[N[S]]$, which means, in other words, that $S \in \Psi(G)$. Finally, we may conclude that $\operatorname{Crown}(G) \subseteq \Psi(G)$.

By Theorem 2.2. each critical independent is also a crown. The converse is not generally true. For instance, if $K_{n, 1}=(A, B, E)$ has $|A|=n \geq 2$, then every proper subset $S$ of $A$ is a crown but is not critical, because $d(S)=|S|-1<d(A)=n-1$. Notice that $A$ is both a maximum critical independent set and a maximum crown for $K_{n, 1}$. 
Corollary 2.3 [37] Every critical independent set is a local maximum independent set as well.

Combining Theorems 1.9 and 2.2. we deduce the following.

Corollary $2.4[8]$ Each critical independent set is included in a maximum independent set.

Corollary $2.5 \operatorname{ker}(G)$ is a crown and, hence, a local maximum independent set.

Combining Theorems 1.5 and 2.2, we get the following.

Corollary 2.6 If $G$ is a König-Egerváry graph, then:

(i) every maximum independent set of $G$ is a crown;

(ii) $\operatorname{core}(G)$ is a crown and, hence, a local maximum independent set.

Corollary 2.7 [1] If $G$ is a graph with a crown $S$, then there is a vertex cover $A$ of $G$ of minimum size that contains all the vertices in $A$ and none of the vertices in $S$.

Proof. By Theorem 2.2, it follows that $S \in \Psi(G)$. Hence, by Theorem 1.9, there is some $B \in \Omega(G)$, such that $S \subseteq B$. Therefore, $A \subseteq V(G)-B$, and this completes the proof, because $V(G)-B$ is a minimum vertex cover of $G$.

Once a crown $S$ is found in a graph $G$, one can remove the vertices of $S \cup N(S)$ and their adjacent edges, to get a smaller graph. In this way, the problem size is now $n_{0}=$ $n-|S|-|N(S)|$, and the parameter size is $k_{0}=k-|H|$.

\section{Critical sets and crowns}

Lemma 3.1 If $A, B \in \operatorname{Crown}(G)$, then there exists a perfect matching between $A \cap N(B)$ and $B \cap N(A)$.

Proof. Consider matchings: $M_{A}$ from $N(A)$ into $A$ and $M_{B}$ from $N(B)$ into $B$. Since $B \cap N(A) \subseteq N(A)$, the matching $M_{A}$ induces an injective mapping $M_{1}$ from $B \cap N(A)$ into $A \cap N(B)$. Similarly, the corresponding restriction of $M_{B}$, say $M_{2}$, maps $A \cap N(B)$ into $B \cap N(A)$. Thus, it follows that $|B \cap N(A)|=|A \cap N(B)|$, and, finally, both $M_{1}$ and $M_{2}$ are bijections.

Theorem 2.2 and Lemma 3.1 imply the following.

Corollary 3.2 [25] If $A, B \in C$ CritIndep $(G)$, then there exists a perfect matching between $A \cap N(B)$ and $B \cap N(A)$.

Remark 3.3 It seems amusing that the matching from Lemma 3.1 exists for so-called side critical independent sets of a bipartite graph [39].

Notice that, according to Theorems [1.5] and 1.2, in a König-Egerváry graph $G$, every $S \in \Omega(G)$ is both a maximum critical independent set and a maximum crown.

Theorem $3.4(V(G)$, Crown $(G))$ is an augmentoid for every graph $G$. 
Proof. Suppose $A$ and $B$ are crowns. Let $A^{1}=A \cup B_{0}$, where $B_{0}=B-N[A]$.

First, we show that $A^{1}$ is a crown, i.e., there is a matching $M^{1}$ from $N\left(A^{1}\right)=N(A) \cup$ $N\left(B_{0}\right)$ into $A^{1}$. Let us define it on the set $A \subseteq A^{1}$ as the matching $M_{A}$ from $N(A)$ into $A$, which exists by definition of a crown. We are left with $N\left(B_{0}\right)-N(A)$ to handle its part in $M^{1}$.

There are no edges between $N\left(B_{0}\right)-N(A)$ and $A \cap B$.

The set $(A \cap N(B)) \cap\left(N\left(B_{0}\right)-N(A)\right)$ is empty, because $B_{0} \cup A$ is independent.

Since $B$ is a crown, there is a matching $M_{B}$ from $N(B)$ into $B$, which maps the set $A \cap N(B)$ onto the set $B \cap N(A)$ (as in the proof of Lemma 3.1). Thus, the restriction $M_{B}^{*}$ of $M_{B}$ to $N\left(B_{0}\right)-N(A)$, maps $N\left(B_{0}\right)-N(A)$ into $B_{0}$. Consequently, the set $M_{A} \cup M_{B}^{*}$ is a matching from $N\left(A^{1}\right)=N(A) \cup N\left(B_{0}\right)$ into $A^{1}$.

Similarly, if $B^{1}=B \cup A_{0}$, with $A_{0}=A-N[B]$, then $B^{1}$ is a crown as well.

Second, we show that $\left|A^{1}\right|=\left|B^{1}\right|$. Indeed,

$$
\begin{aligned}
& A^{1}=A_{0} \cup(A \cap N(B)) \cup(A \cap B) \cup B_{0}, \\
& B^{1}=B_{0} \cup(B \cap N(A)) \cup(B \cap A) \cup A_{0} .
\end{aligned}
$$

Hence, in accordance with Lemma 3.1 we obtain

$$
\begin{gathered}
\left|A^{1}\right|=\left|A_{0}\right|+|A \cap N(B)|+|A \cap B|+\left|B_{0}\right|= \\
=\left|B_{0}\right|+|B \cap N(A)|+|A \cap B|+\left|A_{0}\right|=\left|B^{1}\right|,
\end{gathered}
$$

as required.

Now, Proposition 1.15 and Theorem 3.4 imply the following.

Corollary 3.5 Every crown may be enlarged to a maximum crown.

Actually, if, in the proof of Theorem 3.4 the set $B$ happens to be a maximum crown, we infer that $A_{0}=\emptyset$, and, consequently, $A^{1} \subseteq N[B]$. This leads to the following.

Corollary 3.6 Every crown is included in the closed neighborhood of each maximum crown.

Combining Corollary 3.6 and Theorem 2.2 , we obtain the following.

Corollary 3.7 Every critical set is included in the closed neighborhood of each maximum crown.

Theorem 3.8 If $A, B \in \operatorname{Max} C r o w n(G)$, then both $d(A)=d(B)$ and $N[A]=N[B]$.

Proof. Let $A, B \in \operatorname{Max} \operatorname{Crown}(G), H_{1}=G[N[A]]$ and $H_{2}=G[N[B]]$. According to Corollary 2.1, both $H_{1}$ and $H_{2}$ are König-Egerváry graphs.

By Corollary [3.6, both $A \subseteq N_{G}[B]$ and $B \subseteq N_{G}[A]$. Hence $A \in \Omega\left(H_{2}\right)$ and $B \in \Omega\left(H_{1}\right)$, because $A, B$ are independent and $|A|=|B|$. It follows that

$$
\begin{aligned}
& d_{H_{1}}(A)=|A|-\left|N_{H_{1}}(A)\right|=|A|-\left|N_{G}(A)\right|=d_{G}(A)=d(A) \text { and } \\
& d_{H_{2}}(B)=|B|-\left|N_{H_{2}}(B)\right|=|B|-\left|N_{G}(B)\right|=d_{G}(B)=d(B) .
\end{aligned}
$$

By Theorem 1.5, $d_{H_{1}}(A)=d_{H_{1}}(B)$ and $d_{H_{2}}(B)=d_{H_{2}}(A)$, because $A$ and $B$ are maximum independent sets in both $H_{1}$ and $H_{2}$. 
Since $N_{H_{1}}(B) \subseteq N_{G}(B)$, we get

$$
\begin{gathered}
d(A)=d_{G}(A)=d_{H_{1}}(A)=d_{H_{1}}(B)= \\
|B|-\left|N_{H_{1}}(B)\right| \geq|B|-\left|N_{G}(B)\right|=d_{G}(B)=d(B) .
\end{gathered}
$$

Since $N_{H_{2}}(A) \subseteq N_{G}(A)$, we obtain

$$
\begin{gathered}
d(B)=d_{G}(B)=d_{H_{2}}(B)=d_{H_{2}}(A)= \\
|A|-\left|N_{H_{2}}(A)\right| \geq|A|-\left|N_{G}(A)\right|=d_{G}(A)=d(A) .
\end{gathered}
$$

Consequently, we conclude that $d(A)=d(B)$.

Moreover, we infer that $\left|N_{H_{1}}(B)\right|=\left|N_{G}(B)\right|$, which implies $N_{H_{1}}(B)=N_{G}(B)$. Consequently, we get that

$$
N_{G}[B]=B \cup N_{G}(B)=B \cup N_{H_{1}}(B) \subseteq N_{G}[A] .
$$

Similarly, one can deduce that $N_{G}[A]=A \cup N_{G}(A)=A \cup N_{H_{2}}(A) \subseteq N_{G}[B]$.

Therefore, $N_{G}[A]=N_{G}[B]$.

Lemma 3.9 If $A \subseteq B$ and $B$ is a crown, then $d(A) \leq d(B)$.

Proof. Since $B$ is a crown, there is a matching $M_{B}$ from $N(B)$ into $B$. The matching $M_{B}$ maps $N(B)-N(A)$ into $B-A$. Therefore,

$$
\begin{gathered}
d(B)=|B|-|N(B)|=|A|+|B-A|-(|N(A)|+|N(B)-N(A)|)= \\
=|A|-|N(A)|+|B-A|-|N(B)-N(A)| \geq|A|-|N(A)|=d(A),
\end{gathered}
$$

as required.

Theorem 3.10 $(V(G)$, CritIndep $(G))$ is an augmentoid for every graph $G$.

Proof. Suppose $A$ and $B$ are critical independent sets. Hence they are crowns. By Theorem 3.4. $A^{1}=A \cup(B-N[A])$ and $B^{1}=B \cup(A-N[B])$ are crowns as well and $\left|A^{1}\right|=\left|B^{1}\right|$. Since $A \subseteq A^{1}$, Lemma 3.9 implies that $d(A) \leq d\left(A^{1}\right)$. On the other hand, $d\left(A^{1}\right) \leq d(A)$, because $A$ is critical. Thus $A^{1}, B^{1} \in \operatorname{Crit} \operatorname{Indep}(G)$.

Now, Proposition 1.15 and Theorem 3.10 imply the following.

Corollary 3.11 [26] An inclusion maximal critical independent set is a maximum critical independent set.

Theorem 3.12 MaxCritIndep $(G)=\operatorname{Max} \operatorname{Crown}(G)$.

Proof. Let $A \in$ MaxCritIndep $(G)$ and $B \in \operatorname{Max} C r o w n(G)$. By Theorem 2.2, we have that $A \in \operatorname{Crown}(G)$.

According to Theorem 3.4 there exists some $C \in \operatorname{Max} C r o w n(G)$ such that $A \subseteq C$.

By Lemma 3.9 and Theorem 3.8 , we get

$$
d(G)=d(A) \leq d(C)=d(B) \leq d(G),
$$

which implies $d(C)=d(B)=d(G)$, i.e., $C, B \in C r i t I n d e p(G)$. 
Since $A \subseteq C$ and $A \in M a x C r i t I n d e p(G)$, we get that $A=C \in \operatorname{Max} C r i t I n d e p(G)$. Thus $A \in \operatorname{Max} \operatorname{Crown}(G)$.

Since $C \in \operatorname{Max} C r i t \operatorname{Indep}(G), B \in \operatorname{CritIndep}(G)$ and $|B|=|C|$, it follows that $B \in$ $\operatorname{Max} C r i t \operatorname{Indep}(G)$.

Combining Theorem 2.2, Corollary 3.5, and Theorem 3.12, we get the following.

Corollary 3.13 [25] Every critical independent set is contained in a maximum critical independent set.

Lemma 3.14 If $A \in C r i t I n d e p(G)$, then $\alpha(G)=\alpha(G[N[A]])+\alpha(G[V(G)-N[A]])$.

Proof. By Theorem 2.2, $A \in \operatorname{Crown}(G) \subseteq \Psi(G)$, which means that $G[N[A]]$ is a KönigEgerváry graph with $\alpha(G[N[A]])=|A|$. Theorem[1.9 implies that there exists $S \in \Omega(G)$ such that $A \subseteq S$. Since no edge joins any $a \in A$ to vertices belonging to $S-A$ or to $N(S)-N(A)$, it follows that $\alpha(G[V(G)-N[A])=\alpha(G)-|A|$.

Corollary 3.15 [26] For any graph $G$, there is a unique set $X \subseteq V(G)$ such that

(i) $\alpha(G)=\alpha(G[X])+\alpha(G[V(G)-X])$;

(ii) for every maximum critical independent set $A$ of $G, X=N[A]$;

(iii) $G[X]$ is a König-Egerváry graph.

Proof. Let $A, B \in$ MaxCritIndep $(G)$ and $X=N[B]$.

By Lemma 3.14, we get Part (i).

By Theorem 3.12, $A, B \in \operatorname{MaxCrown}(G)$, and Theorem 3.8 implies $N[A]=N[B]$, i.e., Part (ii) holds.

Since $B \in \operatorname{Max} \operatorname{Crown}(G) \subseteq \operatorname{Crown}(G)$, we have that $G[X]=G[N[B]]$ is a KönigEgerváry graph, which completes the proof.

Butenko and Trukhanov showed that identifying a non-empty critical independent set gives a polynomial-time reduction of the problem of finding a maximum independent set to a proper subgraph. Since a maximum critical independent set may be included in a maximum independent set of a graph, both it and its neighbors may be removed, reducing the problem of finding a maximum independent set to a subgraph. Thus critical independent sets give a polynomially tractable step for the well-known NP-hard problem of finding a maximum independent set in a graph [15]. Moreover, there are parallel algorithms returning maximum critical independent sets [11].

Theorem 3.16 [25] One can compute a maximum critical independent set in polynomial time.

Combining Theorems 3.12 and 3.16, we get the following.

Corollary 3.17 [1] Isolating a crown of maximum order is solvable in polynomial time.

If core $(G)$ is not critical set, then $\operatorname{core}(G)$ is not necessarily a crown. For instance, consider the graph from Figure 5 , whose $\operatorname{core}(G)$ is neither a critical set, nor a crown.

Remark 3.18 If $A, B \in \operatorname{Crown}(G)$, then $A \cap B$ is not necessarily a crown; e.g., $P_{5}=$ $\left(\left\{v_{1}, v_{2}, v_{3}, v_{4}, v_{5}\right\},\left\{v_{1} v_{2}, v_{2} v_{3}, v_{3} v_{4}, v_{4} v_{5}\right\}\right)$ has $\left\{v_{1}, v_{3}\right\},\left\{v_{3}, v_{5}\right\} \in$.Crown $\left(P_{5}\right)$, while $\left\{v_{1}, v_{3}\right\} \cap$ $\left\{v_{3}, v_{5}\right\}=\left\{v_{3}\right\} \notin \operatorname{Crown}\left(P_{5}\right)$. 


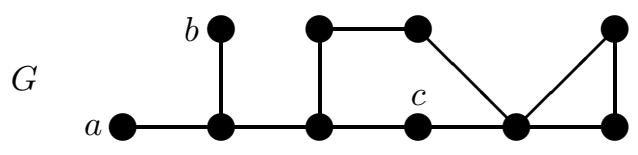

Figure 5: $\operatorname{core}(G)=\{a, b, c\}$ and $d(\operatorname{core}(G))=0<1=d(G)$.

Proposition 3.19 If $A, B \in \operatorname{Crown}(G)$, such that $A \cup B$ is independent, then $A \cup B \in$ Crown $(G)$ as well.

Proof. There exist a matching $M_{1}$ from $N(A)$ into $A$ and a matching $M_{2}$ from $N(B)$ into $B$. Let $M_{3}$ be the restriction of $M_{1}$ to $N(A)-N(B)$. Then, $M_{2} \cup M_{3}$ is a matching from $N(A) \cup N(B)$ into $A \cup B$. Consequently, $A \cup B$ is a crown.

It is worth mentioning that a similar result is true for local maximum independent sets.

Proposition 3.20 [28] If $A$ and $B$ are two disjoint local maximum independent sets in $G$, such that $A \cup B$ is independent, then $A \cup B$ is also a local maximum independent set in $G$.

\section{Crowns and local maximum independent sets}

Notice that there exists a non-bipartite König-Egerváry graph $G$ with $\operatorname{Crown}(G) \neq \Psi(G)$. For instance, the graph $G_{1}$ from Figure 6 is a non-bipartite König-Egerváry graph, $\{d\} \in$ $\operatorname{Crown}\left(G_{1}\right) \cap \Psi\left(G_{1}\right)$, while $\{a\} \in \Psi\left(G_{1}\right)-\operatorname{Crown}\left(G_{1}\right)$.
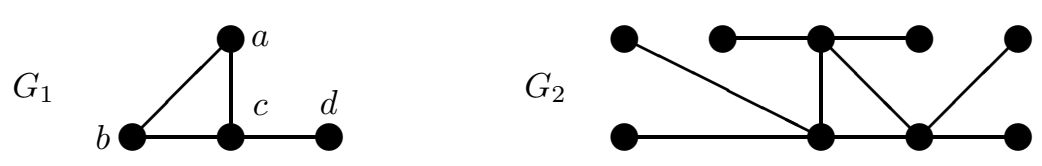

Figure 6: $\operatorname{Crit}\left(G_{1}\right) \neq \operatorname{Crown}\left(G_{1}\right)$ and $\operatorname{Crown}\left(G_{2}\right)=\Psi\left(G_{2}\right)$.

Proposition 4.1 Crown $(G)=\Psi(G)$ if and only if $G[N[S]]$ is a König-Egerváry graph for every $S \in \Psi(G)$. In particular, $G$ is a König-Egerváry graph.

Proof. By Corollary [2.1, $G[N[S]]$ is a König-Egerváry graph for every $S \in \Psi(G)$, because $\Psi(G)=\operatorname{Crown}(G)$.

Conversely, by Theorem 1.1 for each $S \in \Psi(G)$ there is a matching from $N(S)$ to $S$, i.e. $S \in \operatorname{Crown}(G)$.

Since every subgraph of a bipartite graph is bipartite (König-Egerváry), we conclude with the following.

Corollary 4.2 [44] If $G$ is a bipartite graph, then Crown $(G)=\Psi(G)$.

The converse of Corollary 4.2 is not generally true. For instance, the non-bipartite KönigEgerváry graph $G_{2}$ from Figure 6 satisfies $\operatorname{Crown}\left(G_{2}\right)=\Psi\left(G_{2}\right)$.

Combining Theorems 1.12 (i), (ii) and 4.2, we get the following. 
Corollary 4.3 (i) For every tree, Crown $(T)$ is a greedoid.

(ii) If $G$ is a bipartite graph with a unique maximum matching, then $\operatorname{Crown}(G)$ is a greedoid.

The graph $G_{1}=K_{3} \circ K_{1}$ from Figure 7 is not bipartite, and $\operatorname{Crown}(G)=\Psi(G)$. Notice that $K_{3} \circ K_{1}$ is very well-covered.

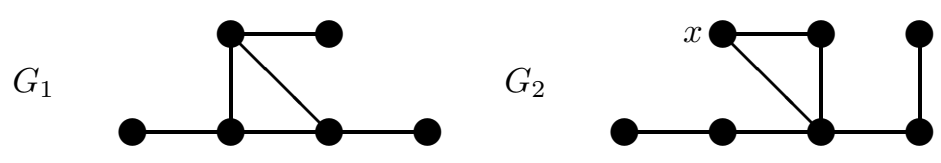

Figure 7: Both $G_{1}$ and $G_{2}$ are well-covered.

Proposition 4.4 If $G$ is a very well-covered graph, then Crown $(G)=\Psi(G)$.

Proof. By Theorem 2.2, it is enough to show that $\Psi(G) \subseteq \operatorname{Crown}(G)$. If $S \in \Psi(G)$, then $S$ is independent and $G[N[S]]$ is a König-Egerváry graph, by Theorem $1.13(i)$. Now, the conclusion follows from Proposition 4.1

Since $H \circ K_{1}$ is very well-covered, for every graph $H$, we infer the following.

Corollary 4.5 If $G=H \circ K_{1}$, then Crown $(G)=\Psi(G)$.

Proposition 4.4 is not true for all well-covered graphs. For instance, the graph $G_{2}$ from Figure 7 is well-covered, and $\{x\} \in \Psi(G)-\operatorname{Crown}(G)$.

Taking into account Theorem 1.13(iii) and Proposition 4.4, we get the following.

Corollary 4.6 If $G$ is a very well-covered graph with a unique maximum matching, then Crown $(G)$ is a greedoid.

There are non-bipartite and non-very well-covered graphs satisfying $\operatorname{Crown}(G) \neq \Psi(G)$; e.g., the graph $G_{2}$ from Figure 6 where $\{x\} \in \Psi(G)-\operatorname{Crown}(G)$.

If $H$ is a König-Egerváry subgraph of a graph $G$, it is not true that there is some maximum independent set $S$ in $H$, such that both $S$ is a crown of $G$ and $G\left[S \cup N_{G}(S)\right]=H$. See, for instance, the subgraphs $H_{1}$ and $H_{2}$ of the graphs $G_{1}$ and $G_{2}$, respectively, from Figure 8 (notice that $G_{1}$ is bipartite, while $G_{2}$ is a non-bipartite König-Egerváry graph).
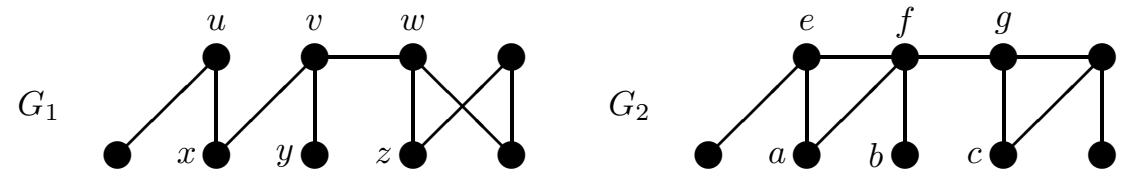

Figure 8: $H_{1}=G_{1}[\{x, y, z, u, v, w\}]$ and $H_{2}=G_{2}[\{a, b, c, e, f, g\}]$ are König-Egerváry graphs.

The definition of a crown and Corollary 2.1 imply the following.

Corollary 4.7 Let $H=S * A$ be a König-Egerváry subgraph of $G$. Then $S \in C r o w n(G)$ if and only if $N_{G}(S)=A$.

Combining Theorems 1.1 1.5, 3.12 we obtain the following. 
Theorem 4.8 These assertions are equivalent:

(i) $G$ is a König-Egerváry graph;

(ii) $\operatorname{Crown}(G) \cap \Omega(G) \neq \emptyset$;

(iii) $\operatorname{Max} \operatorname{Crown}(G) \cap \Omega(G) \neq \emptyset$;

(iv) $\operatorname{Max} \operatorname{Crown}(G)=\Omega(G)$.

Let notice that there exists a tree $G$, such that $\operatorname{CritIndep}(G) \neq \operatorname{Crown}(G)$. For instance, the path $P_{3}=(\{a, b, c\},\{a b, b c\})$ has $\operatorname{CritIndep}\left(P_{3}\right)=\{\{a, c\}\}$, while $\operatorname{Crown}\left(P_{3}\right)=$ $\{\emptyset,\{a\},\{c\},\{a, c\}\}$.

It is easy to see that $\operatorname{CritIndep}\left(C_{2 n+1}\right)=\operatorname{Crown}\left(C_{2 n+1}\right)=\{\emptyset\} \neq \Psi\left(C_{2 n+1}\right)$, while CritIndep $\left(C_{2 n}\right)=\operatorname{Crown}\left(C_{2 n}\right)=\Psi\left(C_{2 n}\right)$.

Proposition 4.9 If $G$ is a König-Egerváry graph with a perfect matching, then CritIndep $(G)=$ $\operatorname{Crown}(G)$.

Proof. By Theorem 1.4, we get $d(G)=\alpha(G)-\mu(G)=0$. According to Theorem 2.2, we have CritIndep $(G) \subseteq \operatorname{Crown}(G)$. Suppose, to the contrary, that there is some $A \in \operatorname{Crown}(G)$ such that $A \notin$ CritIndep $(G)$. It follows that $d(A)=|A|-|N(A)|<0$. Consequently, there is no matching from $N(A)$ into $A$, in contradiction with $A \in C r o w n(G)$.

The converse to Proposition 4.9 is not true. For instance, $\operatorname{CritIndep}\left(C_{5}\right)=\operatorname{Crown}\left(C_{5}\right)$, while $C_{5}$ is not a König-Egerváry graph.

The König-Egerváry graphs $G_{1}, G_{2}$ and $G_{2}$ from Figure 9 have $d\left(G_{1}\right)=d\left(G_{2}\right)=1$, while $d\left(G_{3}\right)=0$. In addition, $\{a\} \in \Psi\left(G_{1}\right)-$ CritIndep $\left(G_{1}\right)$, and $\{u, v\} \in \Psi\left(G_{2}\right)-$ CritIndep $\left(G_{2}\right)$, which means that both CritIndep $\left(G_{1}\right) \neq \Psi\left(G_{1}\right)$ and $\operatorname{CritIndep}\left(G_{2}\right) \neq \Psi\left(G_{2}\right)$.

$G_{1}$

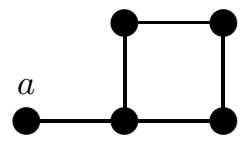

$G_{2}$

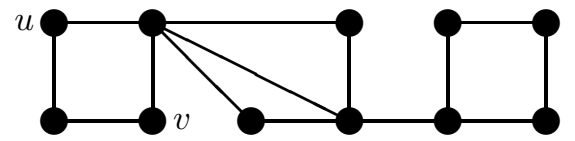

$G_{3}$

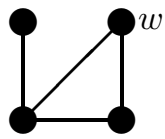

Figure 9: König-Egerváry graphs. Only $G_{3}$ has a perfect matching.

Proposition 4.10 If CritIndep $(G)=\Psi(G)$, then $G$ is a König-Egerváry graph with a perfect matching.

Proof. Since $\Omega(G) \subseteq \Psi(G)$, we infer that every maximum independent set of $G$ is critical. Consequently, by Theorem 1.5, $G$ is a König-Egerváry graph. Further, Theorem 1.4 implies

$$
d(S)=d(G)=\alpha(G)-\mu(G)=d(\emptyset)=0,
$$

for every $S \in \Omega(G)$, because $\emptyset \in \Psi(G)=\operatorname{CritIndep}(G)$. Hence we obtain

$$
\alpha(G)=|S|=|N(S)|=|V(G)-S|=\mu(G),
$$

i.e., $G$ has a perfect matching.

Notice that the converse of Proposition 4.10 is not true, even if $G$ has a unique perfect matching; e.g., consider the graph $G_{3}$ from Figure 9] where $\{w\} \in \Psi\left(G_{3}\right)-\operatorname{CritIndep}\left(G_{3}\right)$.

Combining Propositions 4.10, 4.9 and Theorem 4.2 we conclude with the following. 
Corollary 4.11 Let $G$ be a bipartite graph. Then CritIndep $(G)=\Psi(G)$ if and only if $G$ has a perfect matching.

Corollary 4.11 can not be extended to König-Egerváry graphs. For example, consider the graph $G_{3}$ from Figure 9

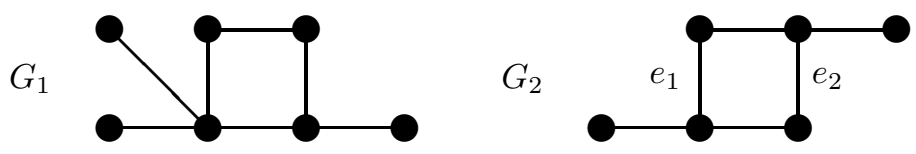

Figure 10: Bipartite graphs with maximum matchings that are uniquely restricted.

Consider the bipartite graphs from Figure 10. According to Theorem 1.12( $($ ii), the family $\Psi\left(G_{1}\right)$ is a greedoid, while $\Psi\left(G_{2}\right)$ is not a greedoid, because the maximum matching $\left\{e_{1}, e_{2}\right\}$ is not uniquely restricted. However, $G_{1}$ has no perfect matchings, and therefore, CritIndep $\left(G_{1}\right) \neq \Psi\left(G_{1}\right)$, by Corollary 4.11 .

Theorem 4.12 Let $G$ be a bipartite graph. Then CritIndep $(G)$ is a greedoid if and only if $G$ has a unique perfect matching.

Proof. By Theorems 1.5 and 4.2 , we infer that

$$
\Omega(G) \subseteq \text { CritIndep }(G) \subseteq \operatorname{Crown}(G)=\Psi(G) .
$$

Hence, every $S \in \Omega(G)$ has an accessibility chain, i.e., there are $S_{k} \in \operatorname{CritIndep}(G)$ such that

$$
\emptyset=S_{0} \subset S_{1} \subset \ldots \subset S_{\alpha(G)}=S \text { and }\left|S_{k}\right|=k .
$$

It follows that $\left|S_{0}\right|=0=\left|N\left(S_{0}\right)\right|$, which, by Theorem 1.4, implies

$$
d(G)=\alpha(G)-\mu(G)=d\left(S_{0}\right)=0 .
$$

Consequently, $G$ has a perfect matching, say $M$. Hence, Corollary4.11implies $\operatorname{CritIndep}(G)=$ $\Psi(G)$. Further, according to Theorem 1.12(ii), all maximum matchings of $G$ must be uniquely restricted. In other words, $M$ is the unique perfect matching of $G$.

The converse follows by combining Theorem 1.12(ii) and Corollary 4.11

Combining Theorems 1.12 (ii) and 4.12, we obtain the following.

Corollary 4.13 If $G$ is a bipartite graph such that $C r i t I n d e p(G)$ is a greedoid, then CritIndep $(G)=$ $\Psi(G)$.

The converse of Corollary 4.13 is not true; e.g., consider $C_{4}$.

Let us notice that $K_{1}$ is a tree and $\operatorname{CritIndep}\left(K_{1}\right) \neq \Psi\left(K_{1}\right)$, since $\emptyset \in \Psi\left(K_{1}\right)-$ CritIndep $\left(K_{1}\right)$. Taking into account that a tree may have at most one perfect matching, we get the following.

Corollary 4.14 Let $T$ be a tree of order at least two. Then the following are equivalent:

(i) $\operatorname{CritIndep}(T)=\operatorname{Crown}(G)$;

(ii) $\operatorname{CritIndep}(T)=\Psi(T)$;

(iii) $d(T)=0$;

(iv) $T$ has a perfect matching;

(v) CritIndep $(T)$ is a greedoid. 
Proof. (i) $\Leftrightarrow$ (ii) It follows by combining Theorems 2.2, 4.2 ,

(ii) $\Rightarrow$ (iii) The tree $T$ has a leaf, say $v$, because its order is at least two. Since, clearly, $\{v\} \in \Psi(T)$, we get that $\{v\} \in C r i t I n d e p(T)$, and hence, $d(T)=d(\{v\})=0$.

(iii) $\Leftrightarrow$ (iv) As a König-Egerváry graph, $T$ satisfies $d(T)=\alpha(T)-\mu(T)$, according to Theorem 1.4. Consequently, we get that

$$
d(T)=0 \Leftrightarrow \alpha(T)=\mu(T) \Leftrightarrow T \text { has a perfect matching. }
$$

(iii) $\Rightarrow$ (ii) By Theorem 2.2, it is enough to show that $\Psi(T) \subseteq \operatorname{CritIndep}(T)$.

Assume, to the contrary, that there is some $S \in \Psi(T)-C r i t I n d e p(T)$. Hence, $d(S)=$ $|S|-|N(S)|<0=d(T)$, i.e., $|S|<|N(S)|$. On the other hand, since $T[S]$ is a forest and $S \in$ $\Omega(T[S])$, we infer that $|S| \geq|N(S)|$, a contradiction. Consequently, $\operatorname{CritIndep}(T)=\Psi(T)$.

(ii) $\Rightarrow(v)$ Clear, as $\Psi(T)$ is a greedoid for every tree $T$.

(v) $\Rightarrow$ (iii) By Theorem 1.5, $\Omega(T) \subseteq$ CritIndep $(T)$. Hence, every $S \in \Omega(T)$ has an accessibility chain, i.e., there are $S_{k} \in \operatorname{Crit} \operatorname{Indep}(T)$ such that $S_{0} \subset S_{1} \subset \ldots \subset S_{\alpha(T)}=S$ and $\left|S_{k}\right|=k$. It follows that $\left|S_{0}\right|=0=\left|N\left(S_{0}\right)\right|$, which implies $d(T)=d\left(S_{0}\right)=0$.

Corollary 4.11 and Corollary 4.2 imply that if $G$ is a bipartite graph, then $\operatorname{CritIndep}(G)=$ $\operatorname{Crown}(G)=\Psi(G)$ if and only $G$ has a perfect matching. For instance, $\operatorname{CritIndep}\left(C_{6}\right)=$ $\Omega\left(C_{6}\right) \cup\{\emptyset\}=\Psi\left(C_{6}\right)$, while CritIndep $\left(C_{5}\right)=\{\emptyset\} \neq \Omega\left(C_{5}\right) \cup\{\emptyset\}=\Psi\left(C_{5}\right)$.

Theorem 4.15 CritIndep $(G)=\operatorname{Crown}(G)=\Psi(G)$ if and only if $G[N[S]]$ is a KönigEgerváry graph with a perfect matching for every $S \in \Psi(G)$. In particular, $G$ is a KönigEgerváry graph with a perfect matching.

Proof. Assume that CritIndep $(G)=\operatorname{Crown}(G)=\Psi(G)$.

By Proposition 4.10, $G$ is a König-Egerváry graph with a perfect matching, say $M$.

By Theorem 1.2, $M \subseteq(A, V(G)-A)$, for every $A \in \Omega(G)$.

Let $S \in \Psi(G)$.

- By Proposition 4.1] $G[N[S]]$ is a König-Egerváry graph. Therefore, by Theorem 1.1(ii), there is a matching from $N(S)$ into $S$. Hence, $|N(S)| \leq|S|$.

- By Theorem [1.9, there is some $B \in \Omega(G)$ such that $S \subseteq B$. Consequently, the trace of $M$ on $G[N[S]]$ is a matching from $S$ into $N(S)$. Thus $|S| \leq|N(S)|$.

- All in all, $|S|=|N(S)|$. In other words, the trace of $M$ on $G[N[S]]$ must be a perfect matching.

Conversely, by Proposition 4.9 , we have that $\operatorname{CritIndep}(G)=\operatorname{Crown}(G)$, and by Proposition 4.1, we get that $\operatorname{Crown}(G)=\Psi(G)$.

For example, the graph $G$ from Figure 11 is a König-Egerváry graph, $S=\{a, b, c, d\} \in$ $\Psi(G)$ and $|S| \geq|N(S)|$, but $S \notin \operatorname{Crown}(G)$. However, $\{c, d\} \in \Psi(G)$ and $|\{c, d\}|<$ $|N(\{c, d\})|=\mid\{u, v, w\}) \mid$, but again $\{c, d\} \notin \operatorname{Crown}(G)$.

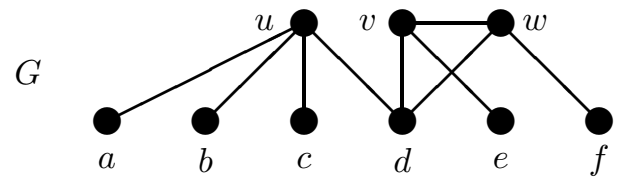

Figure 11: $G$ is a König-Egerváry graph with $\Omega(G)=\{\{a, b, c, d, e, f\}\}$.

By Theorem 1.12(iii) and Theorem 4.15, we obtain the following. 
Corollary 4.16 If $G$ is a triangle-free graph with a unique perfect matching, and $G[N[S]]$ is a König-Egerváry graph with a perfect matching for every $S \in \Psi(G)$, then CritIndep $(G)$ is a greedoid.

\section{Conclusions}

If $\operatorname{CritIndep}(G)=\operatorname{Crown}(G)$, then $d(G)=0$, because $\emptyset \in \operatorname{Crown}(G)$.

Problem 5.1 Characterize graphs satisfying CritIndep $(G)=\operatorname{Crown}(G)$.

Lemma 1.10. Theorem 4.12 and Corollary 4.16 motivate the following.

Conjecture 5.2 Let $G$ be a triangle-free graph. CritIndep $(G)$ is a greedoid if and only if $G[N[S]]$ is a König-Egerváry graph with a unique perfect matching for every $S \in \Psi(G)$.

Theorem 5.3 If $S_{1}, S_{2} \in \Psi(G)$ and $N\left[S_{1}\right] \subseteq N\left[S_{2}\right]$, then there exist $S_{3} \subseteq S_{2}-S_{1}$, such that $S_{1} \cup S_{3} \in \Psi(G)$ and $\left|S_{1} \cup S_{3}\right|=\left|S_{2}\right|$.

Proof. Since $S_{1} \in \Psi(G)$, it follows that $\left|S_{2} \cap N\left[S_{1}\right]\right| \leq\left|S_{1}\right|$. Consequently, $S_{3}=S_{2}-$ $\left(S_{2} \cap N\left[S_{1}\right]\right)$ is independent and $\left|S_{3}\right| \geq\left|S_{2}\right|-\left|S_{1}\right|$. Hence, we get that $S_{1} \cup S_{3}$ is independent. Moreover,

$$
\left|S_{2}\right| \geq\left|S_{1} \cup S_{3}\right|=\left|S_{1}\right|+\left|S_{3}\right| \geq\left|S_{1}\right|+\left|S_{2}\right|-\left|S_{1}\right|=\left|S_{2}\right|,
$$

because $N\left[S_{1}\right] \subseteq N\left[S_{2}\right]$.

Therefore, $\left|S_{2}\right|=\left|S_{1} \cup S_{3}\right|$. In addition, $S_{1} \cup S_{3} \in \Psi(G)$ follows from the fact that $N\left[S_{1} \cup S_{3}\right] \subseteq N\left[S_{2}\right]$.

According to Theorem [1.9] every $A \in \Psi(G)$ can be enlarged to some maximum independent set. By Theorem [5.3. for each $S \in \Omega(G)$ this enlargement can be implemented using only elements of $S$.

Corollary 5.4 [28, 48] (Augmentation version of Nemhauser's and Trotter's Theorem). If $S_{1} \in \Psi(G)$ and $S_{2} \in \Omega(G)$, then there exists $S_{3} \subseteq S_{2}-S_{1}$ such that $S_{1} \cup S_{3} \in \Omega(G)$.

This motivates the following.

Problem 5.5 Characterize graphs whose families of local maximum independent sets are augmentoids.

\section{References}

[1] F. N. Abu-Khzam, M. R. Fellows, M. A. Langston, W. H. Suters, Crown structures for vertex cover kernelization, Theory of Computing Systems 41 (2007) 411-430.

[2] A. A. Ageev, On finding critical independent and vertex sets, SIAM Journal on Discrete Mathematics 7 (1994) 293-295.

[3] N. V. Bagotskaya, V. E. Levit, I. S. Losev, A combinatorial structure ensuring applicability of the dynamic programming method, Automation \& Remote Control 10 (1990) 131-139. 
[4] A. Björner, G. M. Ziegler, Introduction to greedoids, in N. White (ed.), Matroid Applications, 284-357, Cambridge University Press, 1992.

[5] F. Bonomo, M. C. Dourado, G. Durán, L. Faria, L. N. Grippo, M. D. Safe, Forbidden subgraphs and the König-Egerváry property, Discrete Applied Mathematics Volume 161 (2013) 2380-2388.

[6] E. Boros, M. C. Golumbic, V. E. Levit, On the number of vertices belonging to all maximum stable sets of a graph, Discrete Applied Mathematics 124 (2002) 17-25.

[7] E. A. Boyd, U. Faigle, An algorithmic characterization of antimatroids, Discrete Applied Mathematics 28 (1990) 197-205.

[8] S. Butenko, S. Trukhanov, Using critical sets to solve the maximum independent set problem, Operations Research Letters 35 (2007) 519-524.

[9] M. Chlebík, J. Chlebíková, Crown reductions for the minimum weighted vertex cover problem, Discrete Applied Mathematics 156 (2008) 292-312.

[10] E. DeLaVina, Written on the Wall II, Conjectures of Graffiti.pc, http://cms.dt.uh.edu/faculty/delavinae/research/wowII/

[11] E. DeLaVina, C. E. Larson, A parallel algorithm for computing the critical independence number and related sets, Ars Mathematica Contemporanea 6 (2013) 237-245.

[12] R. W. Deming, Independence numbers of graphs - an extension of the König-Egerváry theorem, Discrete Mathematics 27 (1979) 23-33.

[13] R. G. Downey, M. R. Fellows, Parameterized complexity, Springer-Verlag, 1999.

[14] O. Favaron, Very well-covered graphs, Discrete Mathematics 42 (1982) 177-187.

[15] M. Garey, D. Johnson, Computers and intractability, W. H. Freeman and Company, New York, 1979.

[16] O. Goecke, A greedy algorithm for hereditary set systems and a generalization of the Rado-Edmonds characterization of matroids, Discrete Applied Mathematics 20 (1988) $39-49$.

[17] M. C. Golumbic, T. Hirst, M. Lewenstein, Uniquely restricted matchings, Algorithmica 31 (2001) 139-154.

[18] P. L. Hammer, P. Hansen, B. Simeone, Vertices belonging to all or to no maximum stable sets of a graph, SIAM Journal of Algebraic Discrete Methods 3 (1982) 511-522.

[19] R. E. Jamison, Alternating Whitney sums and matchings in trees, Part 1, Discrete Mathematics 67 (1987) 177-189.

[20] A. Jarden, V. E. Levit, E. Mandrescu, Two more characterizations of König-Egerváry graphs, Discrete Applied Mathematics 231 (2017) 175-180.

[21] A. Jarden, V. E. Levit, E. Mandrescu, Critical and maximum independent sets of a graph, Discrete Applied Mathematics 247 (2018) 127-134. 
[22] Y. Kempner, V. E. Levit, Correspondence between two antimatroid algorithmic characterizations, The Electronic Journal of Combinatorics 10 (2003) \#R44.

[23] E. Korach, T. Nguyen, B. Peis, Subgraph characterization of red/blue-split graphs and König-Egerváry graphs, in: Proceedings of the Seventeenth Annual ACM-SIAM Symposium on Discrete Algorithms, ACM Press, 2006, 842-850.

[24] B. Korte, L. Lovász, R. Schrader, Greedoids, Springer-Verlag, Berlin, 1991.

[25] C. E. Larson, A note on critical independence reductions, Bulletin of the Institute of Combinatorics and its Applications 5 (2007) 34-46.

[26] C. E. Larson, The critical independence number and an independence decomposition, European Journal of Combinatorics 32 (2011) 294-300.

[27] V. E. Levit, E. Mandrescu, Combinatorial properties of the family of maximum stable sets of a graph, Discrete Applied Mathematics 117 (2002) 149-161.

[28] V. E. Levit, E. Mandrescu, A new greedoid: the family of local maximum stable sets of a forest, Discrete Applied Mathematics 124 (2002) 91-101.

[29] V. E. Levit, E. Mandrescu, On $\alpha^{+}$-stable König-Egerváry graphs, Discrete Mathematics 263 (2003) 179-190.

[30] V. E. Levit, E. Mandrescu, Local maximum stable sets in bipartite graphs with uniquely restricted maximum matchings, Discrete Applied Mathematics 132 (2004) 163-174.

[31] V. E. Levit, E. Mandrescu, On $\alpha$-critical edges in König-Egerváry graphs, Discrete Mathematics 306 (2006) 1684-1693.

[32] V. E. Levit, E. Mandrescu, Some structural properties of very well-covered graphs, Congressus Numerantium 186 (2007) 97-106.

[33] V. E. Levit, E. Mandrescu, Triangle-free graphs with uniquely restricted maximum matchings and their corresponding greedoids, Discrete Applied Mathematics 155 (2007) 24142425 .

[34] V. E. Levit, E. Mandrescu, An algorithm computing the core of a König-Egerváry graph, 2011, arXiv:1102.1141 [cs.DM], 8 pp.

[35] V. E. Levit, E. Mandrescu, Local maximum stable set greedoids stemming from very well-covered graphs, Discrete Applied Mathematics 160 (2012) 1864-1871.

[36] V. E. Levit, E. Mandrescu, Vertices belonging to all critical independent sets of a graph, SIAM Journal on Discrete Mathematics 26 (2012) 399-403.

[37] V. E. Levit, E. Mandrescu, Critical independent sets and König-Egerváry graphs, Graphs and Combinatorics 28 (2012) 243-250.

[38] V. E. Levit, E. Mandrescu, On the core of a unicyclic graph, Ars Mathematica Contemporanea 5 (2012) 321-327. 
[39] V. E. Levit, E. Mandrescu, Critical sets in bipartite graphs, Annals of Combinatorics 17 (2013) 543-548.

[40] V. E. Levit, E. Mandrescu, On the structure of the minimum critical independent set of a graph, Discrete Mathematics 313 (2013) 605-610.

[41] V. E. Levit, E. Mandrescu, On maximum matchings in König-Egerváry graphs, Discrete Applied Mathematics 161 (2013) 1635-1638.

[42] V. E. Levit, E. Mandrescu, A set and collection lemma, The Electronic Journal of Combinatorics 21 (2014) \#P1.40.

[43] V. E. Levit, E. Mandrescu, On the intersection of all critical sets of a unicyclic graph, Discrete Applied Mathematics 162 (2014) 409-414.

[44] V. E. Levit, E. Mandrescu, Crowns in bipartite graphs, Electronic Notes in Discrete Mathematics 54 (2016) 45-50.

[45] V. E. Levit, E. Mandrescu, Critical and maximum independent sets revisited, Lecture Notes in Computer Science 11548 (2019) 1-16.

[46] L. C. Lorentzen, Notes on covering of arcs by nodes in an undirected graph, Technical report ORC 66-16, Operations Research Center, University of California, Berkeley, California, 1966.

[47] L. Lovász, M. D. Plummer, Matching Theory, Annals of Discrete Mathematics 29 (1986) North-Holland.

[48] G. L. Nemhauser, L. E. Trotter, Jr., Vertex packings: structural properties and algorithms, Mathematical Programming 8 (1975) 232-248.

[49] M. D. Plummer, Some covering concepts in graphs, Journal of Combinatorial Theory 8 (1970) 91-98.

[50] A. Schrijver, Combinatorial Optimization, Springer, Berlin, 2003.

[51] V. V. Serganova, N. V. Bagotskaya, V. E. Levit, I. S. Losev, Greedoids and the greedy algorithm, in the book: Information Transmission \& Processing Systems, vol. 2, IPIT, USSR Academy of Science, Moscow, 49-52. (in Russian)

[52] C. Sloper, Techniques in Parameterized Algorithm Design, Ph.D. thesis, Department of Computer Science, University of Bergen, Norway, 2005.

[53] F. Sterboul, A characterization of the graphs in which the transversal number equals the matching number, Journal of Combinatorial Theory B 27 (1979) 228-229.

[54] C. Q. Zhang, Finding critical independent sets and critical vertex subsets are polynomial problems, SIAM Journal on Discrete Mathematics 3 (1990) 431-438. 